\title{
High-latitude Tertiary Migrations of an Exclusively Tropical Clade: Evidence from Malpighiaceae
}

\section{Citation}

Davis, Charles C., Peter W. Fritsch, Charles D. Bell, and Sarah Mathews. 2004. High-latitude tertiary migrations of an exclusively tropical clade: Evidence from Malpighiaceae. International Journal of Plant Sciences 165(4): S107-S121.

\section{Published Version}

http://dx.doi.org/10.1086/383337

\section{Permanent link}

http://nrs.harvard.edu/urn-3:HUL.InstRepos:2710470

\section{Terms of Use}

This article was downloaded from Harvard University's DASH repository, and is made available under the terms and conditions applicable to Other Posted Material, as set forth at http:// nrs.harvard.edu/urn-3:HUL.InstRepos:dash.current.terms-of-use\#LAA

\section{Share Your Story}

The Harvard community has made this article openly available.

Please share how this access benefits you. Submit a story.

\section{Accessibility}


Int. J. Plant Sci. 165(4 Suppl.):S107-S121. 2004.

(c) 2004 by The University of Chicago. All rights reserved.

$1058-5893 / 2004 / 1650 S 4-0008 \$ 15.00$

\title{
HIGH-LATITUDE TERTIARY MIGRATIONS OF AN EXCLUSIVELY TROPICAL CLADE: EVIDENCE FROM MALPIGHIACEAE
}

\author{
Charles C. Davis, ${ }^{1, *}$ Peter W. Fritsch, $†$ Charles D. Bell, $\neq$ and Sarah Mathews§
}

\begin{abstract}
*Department of Ecology and Evolutionary Biology, University of Michigan Herbarium, 3600 Varsity Drive, Ann Arbor, Michigan 48108-2287, U.S.A.; †Department of Botany, California Academy of Sciences, Golden Gate Park, San Francisco, California 94118, U.S.A.; \#Florida Museum of Natural History, Dickinson Hall, University of Florida, Gainesville, Florida 32611, U.S.A.; and §Arnold Arboretum, Harvard University Herbaria, 22 Divinity Avenue, Cambridge, Massachusetts 02138, U.S.A.
\end{abstract}

Explanations of tropical intercontinental disjunctions involving South America and Africa typically invoke vicariance of western Gondwanan biotas or long-distance dispersal. However, many plant groups originated and diversified well after the last direct connection between Africa and South America (ca. 105 million years before the present [mybp]), and it is unlikely that long-distance dispersal accounts for the distribution of all of these. A less commonly invoked explanation, the boreotropics hypothesis, indicates that some tropical disjunctions arose during the Tertiary via high-latitude land connections when northern forests supported tropical vegetation. Malpighiaceae are widely distributed across Africa and South America and have been explained as ancient "Gondwanian aborigines" (i.e., vicariants of western Gondwanan biotas) or more recent "American colonists" (i.e., long-distance dispersalists from South America into the Old World). Fossil and phylogenetic evidence from clock-independent estimates of molecular divergence times indicate that Malpighiaceae originated in South America during the latest Cretaceous (ca. 68 mybp), in isolation from Africa, and that six amphi-Atlantic disjunctions within the family occurred during three major episodes: late Paleocene (ca. 60 mybp), latest Eocene-earliest Oligocene (ca. 34-31 mybp), and early Miocene (ca. 21-17 mybp). These age estimates reject a Gondwanan origin for Malpighiaceae, and strict dispersal scenarios ignore paleoclimate, paleoland configurations, and fossil evidence that indicates that the family once inhabited northern latitudes. Instead, these data suggest that Paleocene-Oligocene amphi-Atlantic disjunct groups in Malpighiaceae moved into North America from South America via the Caribbean Basin, crossed the North Atlantic into Eurasia, and subsequently reached the Old World Tropics during warm intervals when land configurations would have facilitated this migration. Whether Miocene migrations of evergreen thermophilic Malpighiaceae proceeded via northern latitudes or long-distance dispersal is less clear.

Keywords: Africa, biogeography, long-distance dispersal, nonparametric rate smoothing, penalized likelihood, South America, vicariance, western Gondwana.

\section{Introduction}

Numerous tropical flowering plant lineages have disjunct distributions in Africa (including Madagascar) and South America, and many of these also include a minor Asian component (Thorne 1972). Vicariance of western Gondwanan biotas is often invoked to explain these tropical intercontinental disjunctions (Raven and Axelrod 1974; Gentry 1982, 1993). For example, on the basis of fossil and phylogenetic evidence, Doyle and Le Thomas (1997) inferred that many modern genera of Annonaceae originated and diversified in western Gondwana during the Cretaceous, when lineages moved easily between these connected, or nearly connected, land masses (their fig. 3B).

Although vicariance is a plausible scenario for some amphiAtlantic tropical disjunct lineages, it is apparent that many such plant groups originated and diversified well after the last

\footnotetext{
${ }^{1}$ Author for correspondence; e-mail chdavis@umich.edu.
}

Manuscript received August 2003; revised manuscript received December 2003. direct connection between Africa and South America, ca. 105 million years before the present (mybp; Magallón et al. 1999; Magallón and Sanderson 2001; continental breakup sensu McLoughlin 2001; Wikström et al. 2001). Vicariance cannot account for the distribution of these groups. Instead, longdistance dispersal across the southern Atlantic is often invoked as an alternative scenario (Smith 1973; Thorne 1973; Bremer 1993). For example, Rhipsalis baccata (J. S. Muell.) Stearn is a widespread cactus species that occurs throughout the American Tropics, Africa, Madagascar (and adjacent islands), and Ceylon (Thorne 1973; Barthlott 1983; Barthlott and Taylor 1995). The mid-Tertiary origin of Cactaceae (ca. 30 mybp; Hershkovitz and Zimmer 1997; Nyffeler 2002) and the sticky, copious seeds (Barthlott 1983) of this derived species (Nyffeler 2002) suggest that it traveled by long-distance bird dispersal from the New World, where cacti originated, across the Atlantic to the Old World Tropics (Thorne 1973; Barthlott 1983; Gibson and Nobel 1986; Barthlott and Hunt 1993; Nyffeler 2002).

Despite the importance of long-distance dispersal in the distribution of plants, it cannot account for all of the 
amphi-Atlantic tropical disjunctions that do not result from vicariance. Many taxa with very different dispersal capabilities have nearly identical distributions, suggesting that other mechanisms come into play (Tiffney and Manchester 2001). The existence of a tropical-like, or "boreotropical" (Wolfe 1975), flora extending across the high-latitude regions of the Northern Hemisphere during the Tertiary indicates that conditions were amenable to the northerly intercontinental migration of thermophilic lineages. Moreover, a North Atlantic land bridge connecting North America, Greenland, and Europe is thought to have served as an important conduit for plant migration starting in the Paleocene/Eocene, when local climates were warm enough for frost-intolerant lineages to occur in this region. The low-latitude position of the bridge (perhaps to $45^{\circ} \mathrm{N}$ ) would have provided light levels and climatic conditions sufficient for the presence of evergreen lineages (Tiffney 1985a, 1985b; Donoghue et al. 2001; Sanmartín et al. 2001; Tiffney and Manchester 2001; Zachos et al. 2001). Although the North Atlantic has been recognized as a viable corridor for the migration of thermophilic lineages now restricted to tropical regions, including those with African/South American disjuncts (Raven and Axelrod 1974), mounting evidence from paleontological and phylogenetic studies indicates that this corridor may have been far more important than previously realized for the intercontinental migration of such groups (Krutzsch 1989; Lavin and Luckow 1993; Doyle and Le Thomas 1997, their fig. 3C;
Fritsch 1999, 2001; Lavin et al. 2000; Chanderbali et al. 2001; Renner et al. 2001; Davis et al. 2002a, 2002b).

Most of the hypotheses for the biogeographical history of amphi-Atlantic tropical disjuncts have not been tested within a molecular phylogenetic framework. Recent advances in the methods for estimating divergence times in the absence of a molecular clock, and in methods for incorporating fossils into analyses of divergence times (Sanderson 1997, 1998, 2002, 2003), have provided an opportunity to begin testing such hypotheses. Here we apply molecular and fossil data to better understand the history of amphi-Atlantic tropical disjuncts in Malpighiaceae.

Malpighiaceae are a lineage of trees, shrubs, and vines distributed widely across the tropical and subtropical forests and savannas of the New and Old Worlds. Their present distribution, with only two species common to both these areas (perhaps recently introduced by humans from the New World into Africa; Anderson 1990), has invited repeated speculation about their origin and diversification (Raven and Axelrod 1974; Anderson 1979, 1990; Vogel 1990). Vogel (1990) proposed the "Gondwanian aborigine" hypothesis, which attributes the current distribution to vicariance of western Gondwana (fig. 1A). It implies that the family is relatively old and originated before western Gondwana divided, as did disjuncts between New and Old World species. Anderson (1981, 1990) and others (Raven and Axelrod 1974) proposed the "American colonist" hypothesis, which suggests

\section{A) "Gondwanian aborigines"}

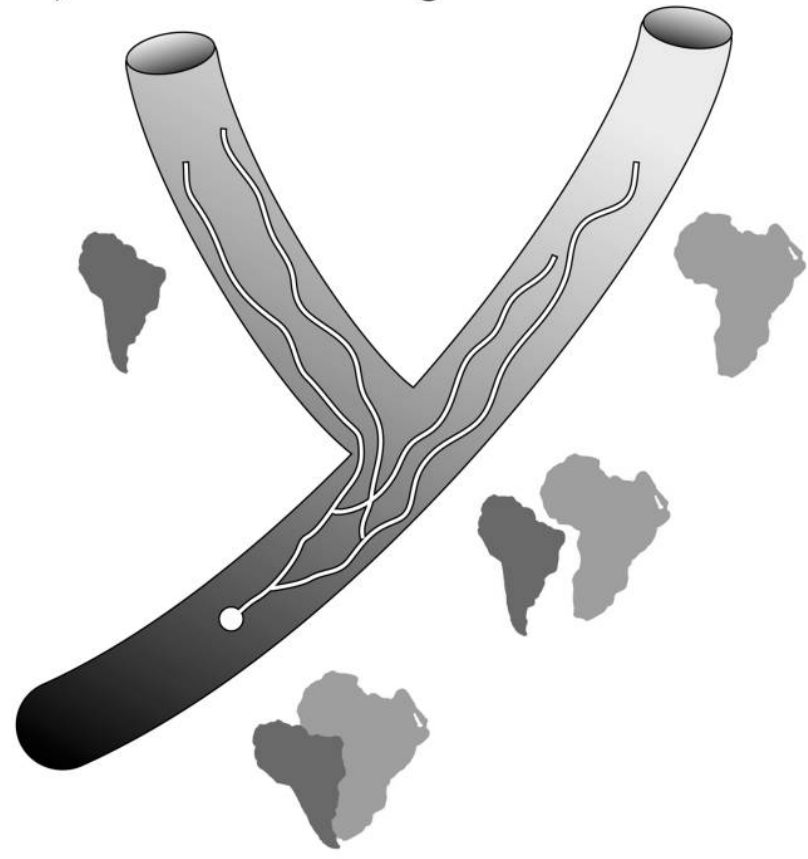

\section{B) "American colonists"}

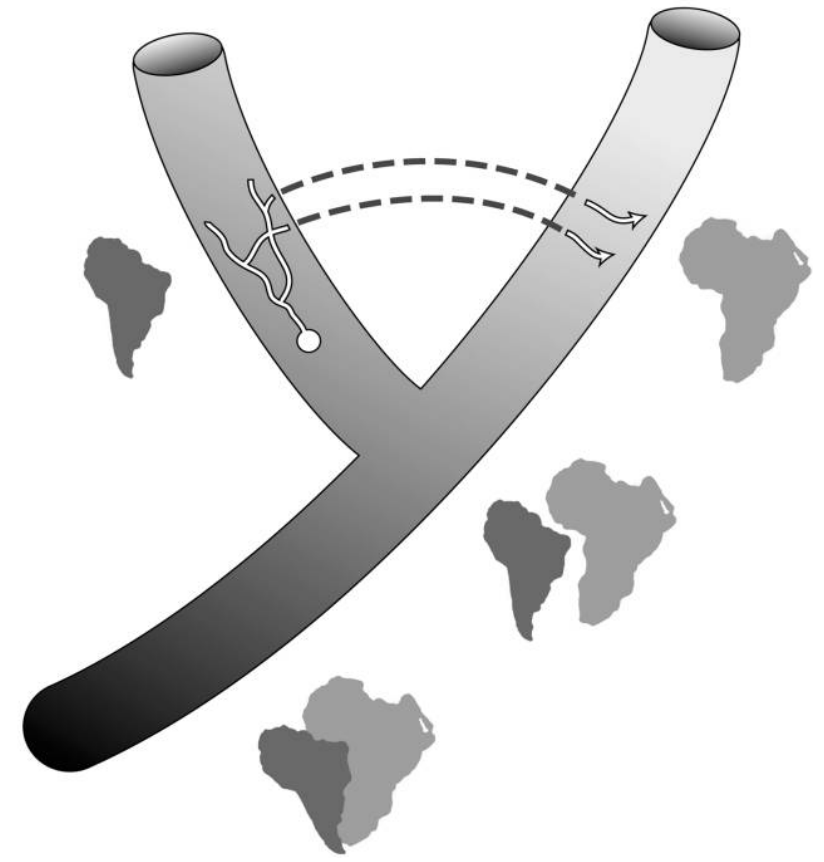

Fig. 1 Gondwanian aborigine hypothesis for Malpighiaceae. A, Origin and diversification of intercontinental disjunctions between Africa and South America are ancient (i.e., at least ca. $105 \mathrm{mybp}$ ) and attributable to continental breakup. Outer (containing) tree corresponds to the breakup of these landmasses and inner (contained) tree to the origin and diversification of disjunct groups. American colonist hypothesis for Malpighiaceae. B, Origin and diversification of intercontinental disjunctions between Africa and South America are attributable to more recent long-distance dispersal from South America across the southern Atlantic Ocean into Africa. Dashed lines indicate transatlantic dispersals. Other disjunct groups for scenario $B$ may have had their origins in Africa and instead dispersed westward. 
that the family is relatively young and originated in northern South America in isolation from Africa and dispersed eastward across the southern Atlantic at least twice (fig. 1B). This hypothesis implies that Malpighiaceae originated after western Gondwana divided and that the divergence times between New and Old World lineages are younger than the most recent connection between South America and Africa. On the basis of fossil and phylogenetic evidence of Malpighiaceae, Davis et al. (2002b) proposed that the family originated in South America, in isolation from Africa, and reached the Old World Tropics via high-latitude North Atlantic land connections during warm climatic intervals, perhaps followed by vicariance through climatic cooling. This North Atlantic corridor may have facilitated the migration of several different lineages of Malpighiaceae throughout the Tertiary (Davis et al. 2002b). This scenario (Davis et al. $2002 b$ ) is similar to Anderson's (1990) hypothesis in that both claim more recent amphi-Atlantic divergence times for Malpighiaceae; Anderson, however, claims long-distance dispersal to explain such disjunctions, whereas Davis et al. (2002b) claim Northern Hemisphere migration followed by vicariance.

To evaluate the biogeographical history of Malpighiaceae, we estimated molecular divergence times across the family using ca. 2000 nucleotides of plastid $n d h \mathrm{~F}$ and nuclear PHYC coding sequence data from 106 species. This data set is similar to that analyzed by Davis et al. (2002b) but includes 35 new taxa and two additional fossil calibration points. One limitation of the study by Davis et al. $(2002 b)$ was its reliance on minimum divergence estimates to test Gondwanan versus alternative scenarios. This study places a maximum age constraint on lineage divergence based on the origin and distribution of the densely sampled eudicot clade, thus incorporating a reliable lower bound on the origin of the family. Also, we contrast estimates of divergence times using both methods, nonparametric rate smoothing (NPRS; Sanderson 1997) and penalized likelihood (PL; Sanderson 2002). PL has been shown (Sanderson 2002) to outperform both clock (Langley and Fitch 1974) and nonclock NPRS methods whenever data depart significantly from a molecular clock.

\section{Material and Methods}

\section{Taxon Sampling}

The PHYC and $n d b \mathrm{~F}$ data sets contain 103 ingroup taxa and three outgroups; 32 PHYC and $28 n d b \mathrm{~F}$ sequences were newly generated for this study (table 1). These data sets include ca. $12 \%$ of the total species diversity of Malpighiaceae, representing 56 of the 66 currently recognized genera (Banisteriopsis, Clonodia, Cordobia, Dinemagonum, Excentradenia, Henleophytum, and Mezia from the New World and Flabellaria, Flabellariopsis, and Digoniopterys from the Old World are not included; see also Davis et al. 2001). Members of the family encompass a range of tropical habitats from lowland rain forest to open savannas.

Within the order Malpighiales (APG 2003), Elatinaceae are strongly placed as sister to Malpighiaceae on the basis of plastid $(r b c \mathrm{~L}$ plus $n d b \mathrm{~F})$ and nuclear $(P H Y C)$ sequence data
(Davis and Chase 2004) and were included here as outgroups. Androstachys johnsonnii (Picrodendraceae) was also included as an additional, more distantly related, outgroup to the Elatinaceae-Malpighiaceae clade (Davis and Chase 2004).

\section{Molecular Methods}

Protocols for extracting DNA, PCR amplification of $n d h \mathrm{~F}$ and $P H Y C$, cloning (for PHYC), and automated sequencing generally followed those reported by Davis et al. (2002a and references cited within; but see also 2001 and 2002b). Nucleotide and amino acid sequences were aligned manually, and the ends were trimmed from each data set to maintain complementarity among taxa. The PHYC and $n d b \mathrm{~F}$ data sets contained 1180 and 810 nucleotides, respectively, before trimming ends.

\section{Phylogenetic Methods}

Data sets were analyzed in combination and included 1830 nucleotides. To assess the level of congruence between these data partitions, we employed the incongruence length difference test (ILD; Farris et al. 1994), implemented in PAUP* version 4.0b10 (Swofford 2002), as the partitionhomogeneity test. We used simple taxon addition (saving 10 trees per replicate) with all uninformative characters excluded, tree bisection-reconnection (TBR) branch swapping, and heuristic searches with 999 repartitions of the data and MulTrees in effect. Gap positions were treated as missing, all characters were weighted equally, and character states were unordered (Fitch parsimony; Fitch 1971). Bootstrap percentages (Felsenstein 1985) for each clade were estimated from 100 heuristic search replicates as above but with simple taxon addition in effect.

A series of hierarchical likelihood ratio tests (Felsenstein 1981; Huelsenbeck and Rannala 1997) was performed with Modeltest version 3.06 (Posada and Crandall 1998) to determine which model of sequence evolution best fit the data. Bayesian analyses were carried out under the preferred model of sequence evolution with Metropolis-coupled Markov chain Monte Carlo $\left(\mathrm{MC}^{3}\right)$ methods implemented in MrBayes version 2.01 (Huelsenbeck and Ronquist 2001). The chain was run for 40 million generations, initiated with a random starting tree, and sampled every 1000 generations; 40,000 trees were sampled from the posterior distribution, which were used to calculate posterior clade probabilities (sample points collected before stationarity, i.e., "burn-in," were eliminated). The incremental heating scheme for each $\mathrm{MC}^{3}$ search used the default settings in MrBayes (three "heated" chains and one "cold" chain). We also used the defaults for the priors on the rate matrix $(0-100)$, branch lengths $(0-10)$, gamma shape parameter $(0-10)$, and the proportion of invariable sites $(0-1)$. A Dirichlet distribution was used for the base frequency parameters, and an uninformative prior was used for the tree topology.

\section{Dating Methods}

We used the single fixed Bayesian tree topology with the lowest likelihood score to test for rate constancy between 
Table 1

Taxa Sequenced with Distribution, Voucher Information, and GenBank (GBAN) Accession Numbers

\begin{tabular}{|c|c|c|c|c|}
\hline & & & Plastid & Nuclear \\
\hline Taxon & Distribution & Voucher & ndhF & PHYC \\
\hline \multicolumn{5}{|l|}{ Elatinaceae $^{\mathrm{a}}$ : } \\
\hline Bergia texana Seub. ex Walp. & NW & Sanders et al. $13525(\mathrm{MICH})$ & GBAN-AY425033 ${ }^{\mathrm{b}}$ & GBAN-AY425091 ${ }^{\mathrm{b}}$ \\
\hline Elatine triandra Schkuhr & NW & Burton et al. $13384(\mathrm{MICH})$ & GBAN-AY425049 ${ }^{\mathrm{b}}$ & GBAN-AY425104 ${ }^{\mathrm{b}}$ \\
\hline \multicolumn{5}{|l|}{ Picrodendraceae $^{\mathrm{a}}$ : } \\
\hline Androstachys johnsonii Prain & OW & Chase $1904(\mathrm{~K})$ & GBAN-AF500495 & GBAN-AF500522 \\
\hline \multicolumn{5}{|l|}{ Malpighiaceae: } \\
\hline \multicolumn{5}{|l|}{ Acmanthera latifolia (Adr. Juss.) } \\
\hline Griseb. in Mart. & NW & Anderson $13771(\mathrm{MICH})$ & GBAN-AF351013 ${ }^{\mathrm{d}}$ & GBAN-AF500528 $8^{\mathrm{c}}$ \\
\hline \multicolumn{5}{|l|}{ Acridocarpus adenophorus } \\
\hline Adr. Juss. & OW & SMG 3399 & GBAN-AY137243 ${ }^{\mathrm{e}}$ & GBAN-AY499049 \\
\hline Acridocarpus chevalieri Sprague & OW & Davis $99-15(\mathrm{~A})$ & GBAN-AY137247 & GBAN-AY499050 \\
\hline Acridocarpus excelsus Adr. Juss. & OW & Rakotomalaza et al. $378(\mathrm{MO})$ & GBAN-AY137248 & GBAN-AY499051 \\
\hline Acridocarpus macrocalyx Engl. & OW & Davis $99-09(\mathrm{~A})$ & GBAN-AF351017 & GBAN-AF500532 \\
\hline \multicolumn{5}{|l|}{ Acridocarpus natalitius } \\
\hline Adr. Juss. & OW & Goldblatt s.n. (PRE) & GBAN-AF351016 ${ }^{\mathrm{d}}$ & GBAN-AF500531 ${ }^{\mathrm{c}}$ \\
\hline \multicolumn{4}{|l|}{ Acridocarpus smeathmannii (DC.) } & GBAN-AY499052 \\
\hline Guill. \& Perr. in Guill. et al. & OW & Davis $99-13(\mathrm{~A})$ & GBAN-AY137261 ${ }^{\mathrm{e}}$ & GBAN-AY499053 \\
\hline \multicolumn{5}{|l|}{ Acridocarpus zanzibaricus } \\
\hline Adr. Juss. & OW & Davis $99-23(\mathrm{~A})$ & GBAN-AY137264 ${ }^{\mathrm{e}}$ & GBAN-AY499054 \\
\hline \multicolumn{5}{|l|}{ Aspicarpa brevipes (DC.) } \\
\hline W. R. Anderson & NW & Cházaro 6295 (MICH) & GBAN-AF351082 ${ }^{\mathrm{d}}$ & GBAN-AF500581 ${ }^{\mathrm{c}}$ \\
\hline Aspicarpa hirtella Rich. & NW & Anderson $13525(\mathrm{MICH})$ & GBAN-AF351033 ${ }^{\mathrm{d}}$ & GBAN-AF500541 ${ }^{\mathrm{c}}$ \\
\hline \multicolumn{5}{|l|}{ Aspidopterys elliptica (Blume) } \\
\hline Adr. Juss. & OW & XVI.G.110 (BO) & GBAN-AF351019 & GBAN-AF436796 ${ }^{\mathrm{f}}$ \\
\hline \multicolumn{5}{|l|}{ Barnebya dispar (Griseb. in Mart.) } \\
\hline W. R. Anderson \& B. Gates & NW & Leoni $1473(\mathrm{MICH})$ & GBAN-AF351073 ${ }^{\mathrm{d}}$ & GBAN-AF500574 ${ }^{\mathrm{c}}$ \\
\hline \multicolumn{5}{|l|}{ Blepharandra heteropetala } \\
\hline W. R. Anderson & NW & Anderson $13782(\mathrm{MICH})$ & GBAN-AF351008d & GBAN-AF500523 \\
\hline Brachylophon curtisii Oliv. & OW & Middleton et al. 387 (A) & GBAN-AF351018 & GBAN-AY137337 \\
\hline Bunchosia armeniaca (Cav.) DC. & NW & FTG77-999C (FTG) & GBAN-AF351045 & GBAN-AF500550' \\
\hline \multicolumn{5}{|l|}{ Burdachia sphaerocarpa } \\
\hline Adr. Juss. & NW & Anderson $13774(\mathrm{MICH})$ & GBAN-AF351071 ${ }^{\mathrm{d}}$ & GBAN-AF500572 \\
\hline Byrsonima crassifolia (L.) H. B. K. & NW & FTG81-680A $(\mathrm{MICH})$ & GBAN-AF351011 ${ }^{\mathrm{d}}$ & GBAN-AF500526 ${ }^{\mathrm{C}}$ \\
\hline \multicolumn{5}{|l|}{ Callaeum septentrionale (Adr. } \\
\hline Juss.) D. M. Johnson & NW & Anderson $13491(\mathrm{MICH})$ & GBAN-AF351058 ${ }^{\mathrm{d}}$ & GBAN-AF500562 ${ }^{\mathrm{c}}$ \\
\hline Camarea axillaris A. St.-Hil. & NW & Amorim 3659 (CEPEC) & GBAN-AY499081 & GBAN-AY499055 \\
\hline \multicolumn{5}{|l|}{ Caucanthus auriculatus (Radlk.) } \\
\hline Nied. & OW & Knox $2128(\mathrm{MICH})$ & GBAN-AF351020d & GBAN-AF436797f \\
\hline Coleostachys genipifolia Adr. Juss. & NW & Anderson $13752(\mathrm{MICH})$ & GBAN-AF351012 ${ }^{\mathrm{d}}$ & GBAN-AF500527 \\
\hline \multicolumn{5}{|l|}{ Diacidia ferruginea (Maguire } \\
\hline \& K. D. Phelps) W. R. Anderson & NW & Berry et al. 4987 (MICH) & GBAN-AF351010 ${ }^{\mathrm{d}}$ & GBAN-AF500525c \\
\hline Dicella nucifera Chodat & NW & Anderson 13607 (MICH) & GBAN-AF351048 & GBAN-AF500553 ${ }^{\mathrm{c}}$ \\
\hline Dinemandra ericoides Adr. Juss. & NW & Dillon \& Teillier $5103(\mathrm{MICH})$ & GBAN-AF351069 ${ }^{d}$ & GBAN-AF500570 \\
\hline \multicolumn{5}{|l|}{ Diplopterys cabrerana (Cuatrec.) } \\
\hline B. Gates & NW & Burnham $1774(\mathrm{MICH})$ & GBAN-AF351039 ${ }^{\mathrm{d}}$ & GBAN-AF500582 ${ }^{\mathrm{c}}$ \\
\hline Echinopterys eglandulosa & & & & \\
\hline (Adr. Juss.) Small & NW & Van Devender 98-178 (MICH) & GBAN-AF351047 ${ }^{\mathrm{d}}$ & GBAN-AF500552 \\
\hline Ectopopterys soejartoi & & & & \\
\hline W. R. Anderson & NW & Callejas 11806 (MICH) & GBAN-AF351064 ${ }^{\mathrm{d}}$ & GBAN-AF500565 \\
\hline Gallardoa fischeri Hicken & NW & Anderson $13580(\mathrm{MICH})$ & GBAN-AF351035 & GBAN-AF500543 ${ }^{\mathrm{c}}$ \\
\hline Galphimia gracilis Bartl. & NW & FTG79-235 (FTG) & GBAN-AF351015 & GBAN-AF500530 c \\
\hline Gaudichaudia albida Schlechtend. & & & & \\
\hline \& Cham. & NW & Anderson 13309A (MICH) & GBAN-AF351034 ${ }^{\mathrm{d}}$ & GBAN-AF500542 \\
\hline Gaudichaudia mcvaughii & & & & \\
\hline W. R. Anderson & NW & Anderson 12699C (MICH) & GBAN-AF351032d & GBAN-AF500540 c \\
\hline Glandonia macrocarpa Griseb. & & & & \\
\hline in Mart. & NW & Anderson $13776(\mathrm{MICH})$ & GBAN-AF351072 ${ }^{\mathrm{d}}$ & GBAN-AF500573 ${ }^{\mathrm{c}}$ \\
\hline
\end{tabular}


Table 1

(Continued)

\begin{tabular}{|c|c|c|c|c|}
\hline & & & Plastid & Nuclear \\
\hline Taxon & Distribution & Voucher & ndhF & PHYC \\
\hline \multicolumn{5}{|l|}{ Heladena multiflora (Hook \& Arn.) } \\
\hline Nied. & NW & Anderson 13599 (MICH) & GBAN-AF351044 ${ }^{\mathrm{d}}$ & GBAN-AF500549 \\
\hline Heteropterys aureosericea Cuatrec. & NW & Burnham 3344 (MICH) & GBAN-AY499082 & GBAN-AY499056 \\
\hline Heteropterys bahiensis Nied. & NW & Amorim 3664 (CEPEC) & GBAN-AY499083 & GBAN-AY499057 \\
\hline \multicolumn{5}{|l|}{ Heteropterys bicolor Adr. Juss. in } \\
\hline A. St.-Hil. & NW & Amorim 2710 (CEPEC) & GBAN-AY499084 & GBAN-AY499058 \\
\hline \multicolumn{5}{|l|}{ Heteropterys byrsonimifolia } \\
\hline Adr. Juss. & NW & Amorim 3663 (CEPEC) & GBAN-AY499085 & GBAN-AY499059 \\
\hline Heteropterys capixaba A. Amorim & NW & Amorim 3317 (CEPEC) & GBAN-AY499086 & GBAN-AY499060 \\
\hline Heteropterys catingarum Adr. Juss. & NW & Souza 455 (CEPEC) & GBAN-AY499087 & GBAN-AY499061 \\
\hline \multicolumn{5}{|l|}{ Heteropterys chrysophylla } \\
\hline (Lam.) DC. & NW & Amorim 3097 (CEPEC) & GBAN-AY499088 & GBAN-AY499062 \\
\hline Heteropterys ciliata Nied. & NW & Anderson 13778 (MICH) & GBAN-AF351049 & GBAN-AF500554 \\
\hline Heteropterys leona (Cav.) Exell & NW \& OW & Davis $99-5(\mathrm{~A})$ & GBAN-AF351050 ${ }^{\mathrm{d}}$ & GBAN-AF500555 \\
\hline Heteropterys lindleyana Adr. Juss. & NW & Amorim 3375 (CEPEC) & GBAN-AY499089 & GBAN-AY499063 \\
\hline Heteropterys megaptera Adr. Juss. & NW & Amorim 3667 (CEPEC) & GBAN-AY499090 & GBAN-AY499064 \\
\hline Heteropterys nitida (Lam.) DC. & NW & Amorim 3327 (CEPEC) & GBAN-AY499091 & GBAN-AY499065 \\
\hline \multicolumn{5}{|l|}{ Heteropterys pauciflora (Adr. Juss. } \\
\hline in A. St.-Hil.) Adr. Juss. & NW & Amorim 3623 (CEPEC) & GBAN-AY499092 & GBAN-AY499066 \\
\hline Heteropterys rhopalifolia Adr. Juss. & NW & Amorim 2879 (CEPEC) & GBAN-AY499093 & GBAN-AY499067 \\
\hline \multicolumn{5}{|l|}{ Heteropterys sanctorum } \\
\hline W. R. Anderson & NW & Amorim 3653 (CEPEC) & GBAN-AY499094 & GBAN-AY499068 \\
\hline \multicolumn{5}{|l|}{ Heteropterys sericea (Cav.) Adr. } \\
\hline Juss. in A. St.-Hil. & NW & Amorim 3342 (CEPEC) & GBAN-AY499095 & GBAN-AY499069 \\
\hline \multicolumn{5}{|l|}{ Heteropterys sincorensis } \\
\hline W. R. Anderson & NW & Queiroz 7145 (CEPEC) & GBAN-AY499096 & GBAN-AY499070 \\
\hline \multicolumn{5}{|l|}{ Heteropterys steyermarkii } \\
\hline W. R. Anderson & NW & Maguire \& Politi $27524(\mathrm{MICH})$ & GBAN-AY499097 & GBAN-AY499071 \\
\hline \multicolumn{5}{|l|}{ Heteropterys ternstroemiifolia } \\
\hline Adr. Juss. & NW & Amorim 3619 (CEPEC) & GBAN-AY499098 & GBAN-AY499072 \\
\hline Heteropterys trichanthera Adr. Juss. & NW & Amorim 3019 (CEPEC) & GBAN-AY499099 & GBAN-AY499073 \\
\hline Hiptage benghalensis (L.) Kurz & OW & Herbst $9221(\mathrm{MICH})$ & GBAN-AF351057 & GBAN-AF500561 ${ }^{\mathrm{c}}$ \\
\hline Hiraea fagifolia (DC.) Adr. Juss. & NW & Anderson $13593(\mathrm{MICH})$ & GBAN-AF351060 ${ }^{\mathrm{d}}$ & GBAN-AF500564 ${ }^{\mathrm{C}}$ \\
\hline \multicolumn{5}{|l|}{ Janusia anisandra (Adr. Juss.) } \\
\hline Griseb. in Mart. & NW & Anderson $13694(\mathrm{MICH})$ & GBAN-AF351028 ${ }^{\mathrm{d}}$ & GBAN-AF500536 c \\
\hline Janusia californica Benth. & NW & Anderson 12539 (MICH) & GBAN-AF351031 ${ }^{\mathrm{d}}$ & GBAN-AF500539 \\
\hline Janusia linearis Wiggins & NW & Anderson $12540(\mathrm{MICH})$ & GBAN-AF351030 ${ }^{\mathrm{d}}$ & GBAN-AF500538 \\
\hline \multicolumn{5}{|l|}{ Janusia mediterranea (Vell.) } \\
\hline W. R. Anderson & NW & Anderson $13686(\mathrm{MICH})$ & GBAN-AF351029d & GBAN-AF500537 \\
\hline Jubelina wilburii W. R. Anderson & NW & Anderson 13789 (MICH) & GBAN-AY499100 & GBAN-AY499074 \\
\hline Lasiocarpus sp. & NW & Anderson $13834(\mathrm{MICH})$ & GBAN-AF351066 ${ }^{\mathrm{d}}$ & GBAN-AF500567 \\
\hline Lophanthera lactescens Ducke & NW & Anderson $13781(\mathrm{MICH})$ & GBAN-AF351009 ${ }^{\mathrm{d}}$ & GBAN-AF500524 ${ }^{\mathrm{c}}$ \\
\hline \multicolumn{5}{|l|}{ Lophopterys floribunda } \\
\hline W. R. Anderson \& C. Cav. Davis & NW & Anderson $13754(\mathrm{MICH})$ & GBAN-AF351078 ${ }^{\mathrm{d}}$ & GBAN-AF500579 \\
\hline \multicolumn{5}{|l|}{ Madagasikaria andersonii } \\
\hline C. Cav. Davis & OW & Davis et al. 20-01 (A) & GBAN-AF436790 f & GBAN-AF436800 f \\
\hline Malpighia emarginata DC. & NW & Anderson 13621 (MICH) & GBAN-AF351023 ${ }^{\mathrm{d}}$ & GBAN-AF436799f \\
\hline Malpighia stevensii W. R. Anderson & NW & Davis $1019(\mathrm{MICH})$ & GBAN-AF351022 ${ }^{\mathrm{d}}$ & GBAN-AF436798 \\
\hline \multicolumn{5}{|l|}{ Mascagnia anisopetala (Adr. Juss. in } \\
\hline A. St.-Hil.) Griseb. in Mart. & NW & Anderson 13598 (MICH) & GBAN-AF351053 ${ }^{\mathrm{d}}$ & GBAN-AF500557 \\
\hline \multicolumn{5}{|l|}{ Mascagnia bracteosa Griseb. in } \\
\hline Mart. & NW & Anderson 13777 (MICH) & GBAN-AF351055 ${ }^{\mathrm{d}}$ & GBAN-AF500559 \\
\hline & NW & Anderson $13710(\mathrm{MICH})$ & GBAN-AF351054 ${ }^{\mathrm{d}}$ & GBAN-AF500558 \\
\hline Mascagnia dipholiphylla (Small) & & & & \\
\hline Bullock & NW & Anderson 13816 (MICH) & GBAN-AF351063 ${ }^{\mathrm{d}}$ & GBAN-AF436795 ${ }^{\mathrm{f}}$ \\
\hline Mascagnia hippocrateoides & & & & \\
\hline (Triana \& Planch.) Nied. & NW & Anderson $13802(\mathrm{MICH})$ & GBAN-AF351059 ${ }^{d}$ & GBAN-AF500563 ${ }^{\mathrm{c}}$ \\
\hline Mascagnia rigida (Adr. Juss. in A. & & & & \\
\hline St.-Hil.) Griseb. in Mart. & NW & Anderson $13691(\mathrm{MICH})$ & GBAN-AY499101 & GBAN-AY499075 \\
\hline
\end{tabular}


Table 1

(Continued)

\begin{tabular}{|c|c|c|c|c|}
\hline & & & Plastid & Nuclear \\
\hline Taxon & Distribution & Voucher & ndhF & PHYC \\
\hline \multicolumn{5}{|l|}{ Mascagnia sepium (Adr. Juss. in } \\
\hline A. St.-Hil.) Griseb. in Mart. & NW & Chase $90160(\mathrm{MICH})$ & GBAN-AF351025 & GBAN-AF436804 ${ }^{\mathrm{f}}$ \\
\hline Mascagnia stannea (Griseb.) Nied. & NW & Anderson $13804(\mathrm{MICH})$ & GBAN-AF351056 ${ }^{\mathrm{d}}$ & GBAN-AF500560 \\
\hline Mcvanghia bahiana W. R. Anderson & NW & Anderson $13690(\mathrm{MICH})$ & GBAN-AF351070 & GBAN-AF500571 ${ }^{\mathrm{c}}$ \\
\hline Microsteira sp. & OW & Davis et al. 38-01 (A) & GBAN-AF436791 ${ }^{\mathrm{f}}$ & GBAN-AF436803 ${ }^{\mathrm{f}}$ \\
\hline Mionandra camareoides Griseb. & NW & Anderson 13585 (MICH) & GBAN-AF351080 & GBAN-AF500580 \\
\hline \multicolumn{5}{|l|}{ Peixotoa glabra Adr. Juss. in } \\
\hline A. St.-Hil. & NW & Anderson $13636(\mathrm{MICH})$ & GBAN-AF351036 ${ }^{\mathrm{d}}$ & GBAN-AF500544 ${ }^{\mathrm{C}}$ \\
\hline Peregrina linearifolia (A. St.-Hil.) & & Krapovickas \& Cristóbal & & \\
\hline W. R. Anderson & NW & $46451(\mathrm{MICH})$ & GBAN-AY499102 & GBAN-AF500535 \\
\hline Philgamia glabrifolia Arènes & OW & Davis $40-01(\mathrm{~A})$ & GBAN-AY499103 & GBAN-AY499076 \\
\hline Pterandra arborea Ducke & NW & Anderson $13766(\mathrm{MICH})$ & GBAN-AF351014 ${ }^{\mathrm{d}}$ & GBAN-AF500529 \\
\hline Ptilochaeta bahiensis Turcz. & NW & Anderson $13725(\mathrm{MICH})$ & GBAN-AF351068 ${ }^{\mathrm{d}}$ & GBAN-AF500569 \\
\hline Ptilochaeta nudipes Griseb. & NW & Anderson $13588(\mathrm{MICH})$ & GBAN-AF351067 ${ }^{\mathrm{d}}$ & GBAN-AF500568 \\
\hline \multicolumn{5}{|l|}{ Rhynchophora phillipsonii } \\
\hline W. R. Anderson & OW & Davis et al. 23b-01 (A) & GBAN-AF436793 ${ }^{\mathrm{f}}$ & GBAN-AF436802 ${ }^{\mathrm{f}}$ \\
\hline \multicolumn{5}{|l|}{ Ryssopterys timoriensis (DC.) Adr. } \\
\hline Juss. in Deless. & OW & XVIII.F.172 (BO) & GBAN-AF351040 & GBAN-AF500545 \\
\hline Spachea correae Cuatrec. \& Croat & NW & Hammel 17746 (MO) & GBAN-AF351074 ${ }^{\mathrm{d}}$ & GBAN-AF500575 \\
\hline Sphedamnocarpus sp. (accession a) & OW & Davis $03-01(\mathrm{~A})$ & GBAN-AY499104 & GBAN-AF500546 ${ }^{\mathrm{C}}$ \\
\hline Sphedamnocarpus sp. (accession b) & OW & Davis $39-01(\mathrm{~A})$ & GBAN-AY499105 & GBAN-AY499077 \\
\hline \multicolumn{5}{|l|}{ Stigmaphyllon paralias Adr. Juss. in } \\
\hline A. St.-Hil. & NW & Anderson $13693(\mathrm{MICH})$ & GBAN-AF351065 & GBAN-AF500566 ${ }^{\mathrm{C}}$ \\
\hline \multicolumn{5}{|l|}{ Stigmaphyllon puberum (Rich.) } \\
\hline Adr. Juss. & NW & Anderson $13793(\mathrm{MICH})$ & GBAN-AF351038 & GBAN-AF436794 \\
\hline \multicolumn{5}{|l|}{ Stigmaphyllon sagraeanum } \\
\hline Adr. Juss. & NW & FTGX-1-6 (FTG) & GBAN-AF351077 & GBAN-AF500578 \\
\hline Tetrapterys discolor (G. Mey.) DC. & NW & Chase $90158(\mathrm{MICH})$ & GBAN-AF351076 ${ }^{\mathrm{d}}$ & GBAN-AF500577 \\
\hline \multicolumn{5}{|l|}{ Tetrapterys microphylla (Adr. Juss. } \\
\hline in A. St.-Hil.) Nied. & NW & Anderson $13644(\mathrm{MICH})$ & GBAN-AF351052 & GBAN-AF500556 \\
\hline Thryallis longifolia Mart. & NW & Anderson $13657(\mathrm{MICH})$ & GBAN-AF351046 ${ }^{\mathrm{d}}$ & GBAN-AF500551 ${ }^{\mathrm{c}}$ \\
\hline Triaspis hypericoides Burch. & OW & Immelman 650 (PRE) & GBAN-AF351021 ${ }^{\mathrm{d}}$ & GBAN-AF500533 ${ }^{\mathrm{c}}$ \\
\hline Tricomaria usillo Hook. \& Arn. & NW & Anderson 13581 (MICH) & GBAN-AF351075 & GBAN-AF500576 ${ }^{\mathrm{c}}$ \\
\hline Triopterys rigida $\mathrm{S}_{\mathrm{w}}$ & NW & Hammel $17816(\mathrm{MICH})$ & GBAN-AF351024 ${ }^{\mathrm{d}}$ & GBAN-AF500534 ${ }^{\mathrm{c}}$ \\
\hline Tristellateia africana S. Moore & OW & Davis $99-25(\mathrm{~A})$ & GBAN-AF351043 ${ }^{\mathrm{d}}$ & GBAN-AF500548 \\
\hline \multicolumn{5}{|l|}{ Tristellateia madagascariensis } \\
\hline Poir. in Lam. & OW & McPherson 14398 (MICH) & GBAN-AF351042 ${ }^{\mathrm{d}}$ & GBAN-AF500547 \\
\hline Tristellateia sp. (accession a) & OW & Davis $19-01(\mathrm{~A})$ & GBAN-AY499106 & GBAN-AY499078 \\
\hline Tristellateia sp. (accession b) & OW & Davis $29-01(\mathrm{~A})$ & GBAN-AY499107 & GBAN-AY499079 \\
\hline Verrucularia glaucophylla Adr. Juss. & NW & Amorim 3662 (CEPEC) & GBAN-AY499108 & GBAN-AY499080 \\
\hline
\end{tabular}

Note. Herbarium acronyms follow Holmgren et al. (1990). Distribution: NW = New World; OW = Old World.

a Outgroups.

b Previously sampled from Davis and Chase (2004).

c Previously sampled from Davis et al. (2002b).

d Previously sampled from Davis et al. (2001).

e Previously sampled from Davis et al. (2002a).

${ }^{f}$ Previously sampled from Davis (2002).

clock versus nonclock trees using $2\left(\ln L_{1}-\ln L_{0}\right)$ as a test statistic. This was compared to a $\chi^{2}$ distribution to assess significance (with $n-2$ degrees of freedom, where $n=$ number of taxa). Because rate constancy was rejected $(P<$ $0.001,-\ln L$ unconstrained $=22,500.89$ vs. constrained $=$ 22,677.55; $\mathrm{df}=104)$, we used clock-independent NPRS (Sanderson 1997) and PL (Sanderson 2002) methods implemented in r8s version 1.6 (Sanderson 2003; http://ginger .ucdavis.edu/r8s) to estimate absolute ages. These methods relax the assumptions of global or local molecular clocks by smoothing changes of nucleotide substitution across the tree. NPRS is a nonparametric method that estimates rates and times by using a least square criterion, which penalizes rapid rate changes across branches in a phylogeny (Sanderson 1997). PL is a semiparametric method that combines the statistical power of parametric methods (models of molecular evolution) with the robustness of nonparametric methods by assigning penalties to limit rate changes between adjacent branches on a phylogeny. Like NPRS, PL assumes that there is an autocorrelation of substitution rates and attempts to 
minimize rate changes between ancestral/descendant branches on a tree (i.e., at the nodes). Branches are allowed to change rates of molecular evolution but are penalized when rates change from ancestral to descendant branches. The crux of the penalized likelihood method is determining the optimal smoothing parameter. The smoothing parameter can vary from very small, in which each branch of the phylogeny has a different substitution rate (saturated model), to very large, in which parameters are essentially clocklike. r8s implements a data-driven cross-validation procedure that systematically prunes terminals from the tree and then estimates parameters from the submatrix under a given smoothing value. It then tries to predict the data for the pruned taxa by using the estimated parameters. Last, it calculates a $\chi^{2}$ error associated with the difference between the predicted and observed data. The optimal smoothing value corresponds to the lowest $\chi^{2}$ error (Sanderson 2002).

NPRS and PL analyses were performed on the single unconstrained Bayesian tree topology mentioned above $(-\ln L=22,500.89$; tree not shown). Branch lengths were estimated for the data set with its best-fitting model. Androstachys was used as the rooting outgroup and was included in the dating analyses. A maximum age of 125 mybp was enforced for the clade (Androstachys, (Elatinaceae, Malpighiaceae)), which corresponds with the probable origin of the densely sampled, and palynologically distinctive, eudicot clade (Magallón et al. 1999) to which Malpighiaceae belong. Our sampling does not correspond directly with all eudicots (Soltis et al. 2003) but rather to the more derived eudicot clade Malpighiales (APG 2003). Malpighiales appear to be very old and began to diversify during the mid-Cretaceous (116-111 million years ago [mya]; C. Davis, unpublished data). The Malpighiales clade should be no older than the first appearance of the distinctive eudicot pollen type. All zero-lengths branches were collapsed before analysis. For the PL analysis, the optimal smoothing parameter, ranging between 0.001 and 10,000 (Sanderson 2003; M. Sanderson, personal communication), was selected before the dating analysis by performing the cross-validation procedure in $\mathrm{r} 8 \mathrm{~s}$.

Absolute age estimates were obtained for nodes 0-6 (fig. 2) by using several calibration points (fig. 2; see details below). These nodes represent the crown age of Malpighiaceae (node 0 ) and the six disjuncts (nodes 1-6) between New and Old World Malpighiaceae (see also Davis et al. 2002b). All age estimates were restarted 10 times to minimize the chance of converging onto a suboptimal solution.

To estimate the $95 \%$ confidence intervals around our age estimates, we used the method developed by Cutler (2000) as implemented in r8s (Sanderson 2003). This procedure uses the curvature of the likelihood surface around the parameter estimate to find the endpoints of optimal age estimates. These values are determined from the decline in log likelihood units around this surface, which is specified in r8s by the cutoff value. Under a normal distribution, a drop in two log likelihood units (cutoff value $=2$ ) corresponds to the $95 \%$ confidence interval (Sanderson 2003). However, as previously noted (Sanderson 2003), there is little reason to believe that this should be the case with real data, and simulation studies (Cutler 2000; Sanderson 2003) have shown that values twice that (i.e., cutoff value $=4$ ) may be necessary. We used a cut- off of four based on these studies. Age estimates for determining confidence intervals were restarted three times to avoid suboptimal solutions.

\section{Fossil Calibration}

A fossil species of Tetrapterys from the early Oligocene (33 mybp; Hably and Manchester 2000) of Hungary and Slovenia provides a reliable minimum age estimate for the stem (see Doyle and Donoghue 1993; Magallón and Sanderson 2001 for stem vs. crown groups) Tetrapterys clade (Davis et al. 2002b; fig. 2, node $A$ ). In addition, we have included two additional fossil calibration points that have not previously been included in any dating analysis of Malpighiaceae.

Eoglandulosa warmanensis Taylor and Crepet was described on the basis of extraordinarily preserved flowering material from the Eocene Claiborne formation of northwestern Tennessee (Taylor and Crepet 1987). This unmistakable malpigh fossil has all of the distinctive features of New World Malpighiaceae flowers, including clawed (or paddleshaped) petals and glandular sepals. Taylor and Crepet (1987) placed this fossil taxon as a member of the New World subfamily Byrsonimoideae (Anderson 1978; Davis et al. 2001, "byrsonimoid" clade) on the basis of the presence of 10 paired and enlarged sepal glands and tricolporate pollen; both are characteristics found in combination only among extant Byrsonimoideae (Anderson 1978; Davis et al. 2001; W. Anderson, personal communication).

There are three major tribes of Byrsonimoideae (Anderson 1978) to which Eoglandulosa may belong: Galphimieae, Byrsonimeae sensu stricto (Davis et al. 2001, i.e., excluding Burdachia, Glandonia, and Mcvaughia), and Acmantherae. With the exception of Galphimia, which has diversified in Mexico, the overwhelming majority of Byrsonimoideae are restricted to South America (Anderson 1978, 1981). It is unlikely that Eoglandulosa belongs with Galphimia (or to other Galphimieae) because most Galphimieae have either very reduced sepal glands (in total number and size) or more often none at all (Anderson 1978). Of the remaining byrsonimoid genera, Byrsonima has a distribution (Anderson 1981) that is most consistent with the distribution (Anderson 1981) and paleoclimate (Dilcher 1973; Frederiksen 1980; Taylor and Crepet 1987) of the Eoglandulosa type locality (Potter and Dilcher 1980; Taylor and Crepet 1987). Species of Byrsonima occur as far north as Florida today (Anderson 1981). In addition, it is the only genus in Byrsonimoideae whose species have an obvious adaptation for widespread migration (Anderson 1978); they have fleshy bird-dispersed fruits, which may have aided their movement out of South America, their likely center of origin (Raven and Axelrod 1974; Anderson 1981, 1990; Davis et al. 2002a). Other possible byrsonimoids have no obvious adaptation for long-distance dispersal; none have winged, setose, or fleshy fruits (Anderson 1978,1981$)$.

Given the proximity and climatic similarity of Eoglandulosa to modern species of Byrsonima and the relative vagility of Byrsonima species, we have placed this fossil taxon as a member of the stem lineage that gave rise to the Byrsonima clade (fig. 2; node B). Biostratigraphic analyses of the Upper Claiborne formation where Eoglandulosa was collected are 


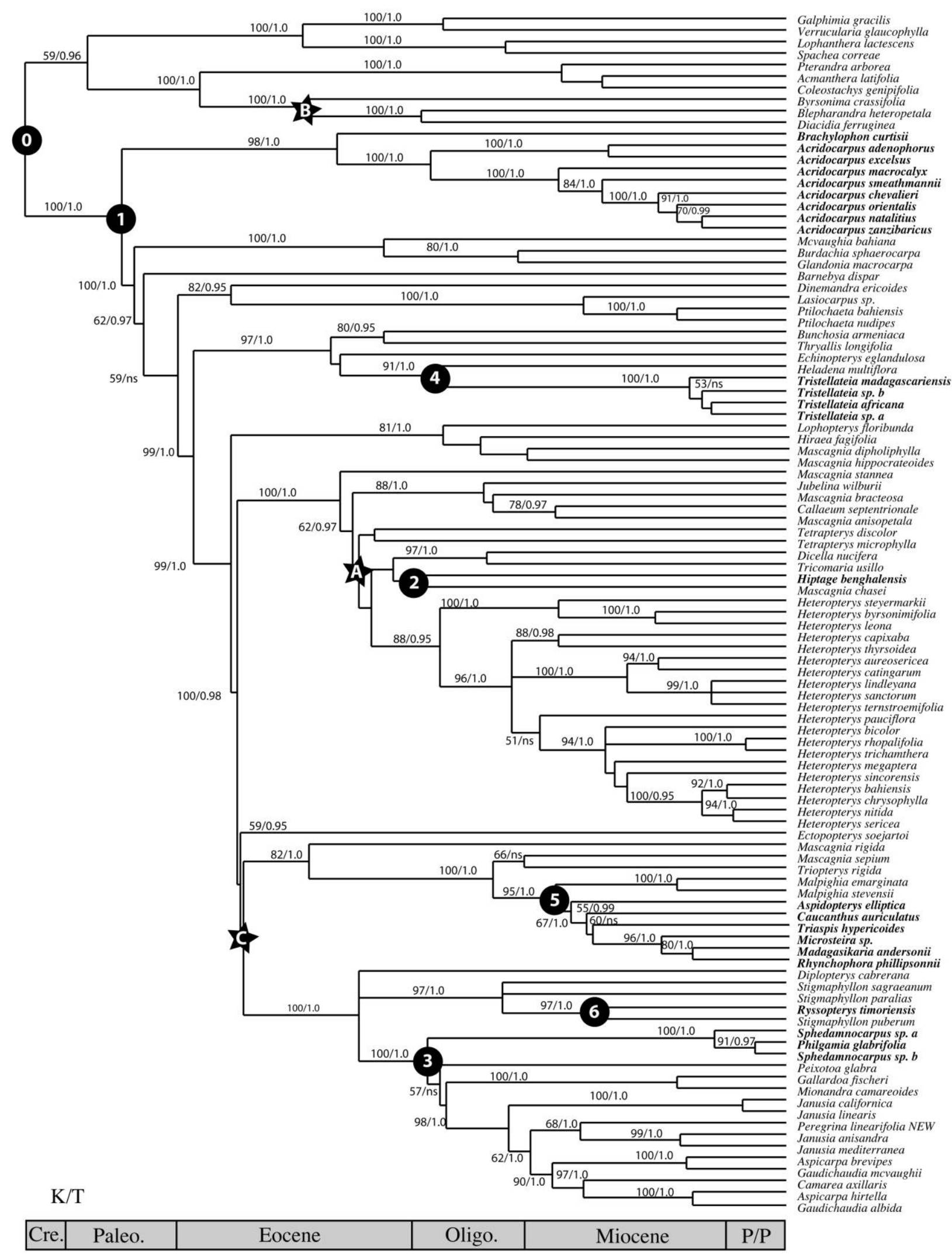

Fig. 2 Penalized likelihood chronogram based on combined $n d b \mathrm{~F}$ and $P H Y C$ data. Fossil calibration points $A, B$, and $C$ are starred, and the seven circled nodes (0-6) correspond to the origin of Malpighiaceae (node 0) and to the six disjunctions between New and Old World clades (nodes 1-6). Old World taxa are shown in boldface. Numbers near nodes are parsimony bootstrap percentages and Bayesian posterior clade probabilities, respectively. See table 2 for confidence intervals around relevant nodes. $\mathrm{P} / \mathrm{P}=$ Pliocene/Pleistocene. 
43-40 mybp in age (Potter and Dilcher 1980; D. Dilcher, personal communication). Because the Warman Pit is one of the oldest beds from this deposit (Potter and Dilcher 1980), we have placed a minimum age on the stem Byrsonima clade (fig. 2; node B) at 43 mybp.

Perisyncolporites pokornyi Germeraad et al. (1968) has a distinctive and mostly hexaporate 12 -rugate pollen grain in which the pores form the apices of an octahedron and the rugae are arranged at the edges of a cube ("banisterioid" type, sensu Lowrie 1982, p. 28, his fig. 3.1). The pores are in or near the ruga, and usually only six of them are porate. The other rugae, which lack pores, connect to those with pores and occur along the equator of the grain. This pollen type is unique among angiosperms (Germeraad et al. 1968) and within Malpighiaceae is synapomorphic for the stigmaphylloid clade (Lowrie 1982; Davis et al. 2001; fig. 2, node C).

Perisyncolporites pokornyi has been widely collected across the tropics by petroleum corporations because it is an important indicator of Paleogene strata, which are rich in hydrocarbon reservoirs (Jaramillo and Dilcher 2001). It has been documented from all major tropical regions from the Eocene, including the Caribbean (Germeraad et al. 1968), South and Central America (Regali et al. 1974; Muller 1981; Rull 1997, 1998; Jaramillo and Dilcher 2001; Jaramillo 2002), Africa (Germeraad et al. 1968; Salard-Cheboldaeff 1991), and Australia (Martin 2002). We fixed the minimum age of the stem stigmaphylloid clade (fig. 2, node C) at ca. 49 mybp (Cenozoic time scale sensu Berggren et al. 1995) on the basis of the oldest reliably dated samples (i.e., outcrop and core) of P. pokornyi from the latest early Eocene of Colombia (Jaramillo and Dilcher 2001; Jaramillo 2002; C. Jaramillo, personal communication). Rull (1997) reported earlier late Paleocene samples of P. pokornyi from Venezuela, but his data are presented as an undistinguished mixture of less reliably dated well-type cuttings and more reliable core samples. Moreover, his data (Rull 1997, his fig. 5) show several other fossil pollen types within the same Paleocene time frame as $P$. pokornyi that are much older than their known occurrences (C. Jaramillo, Instituto Colombiano del Petroleo, personal communication).

We performed the NPRS analyses using several different fossil calibration points, three with each fossil calibration point enforced independently and one where all calibration points were enforced in a single analysis. Age estimates based on single fossil calibration points were performed to assess the degree to which estimated ages corroborate other clade ages for which fossil calibration points were available but not enforced. In the case of the PL analysis, all three calibration points were enforced in a single analysis to estimate absolute ages.

\section{Results}

\section{Phylogenetic Analyses}

The $n d b \mathrm{~F}$ and $P H Y C$ data sets were not significantly different, as determined by the ILD test $(P=0.20)$, and were analyzed in combination. A general time-reversible model (Rodríguez et al. 1990) including parameters for invariant sites and rate variation $(\mathrm{GTR}+\mathrm{I}+\Gamma)$ best fit the data and was used to conduct maximum likelihood analyses in MrBayes. The optimal likelihood tree from the combined data analyses in MrBayes had a log-likelihood score of $-\ln L=22,500.89$ (tree not shown). This tree was topologically similar to previous less densely sampled analyses (Davis et al. 2002b). Six disjunctions were identified between New and Old World lineages (nodes 1-6; fig. 2).

\section{Molecular Dating}

The results of our molecular dating analyses are summarized in table 2 . When fossil calibration points for nodes A and $B$ were enforced independently by using NPRS (table $2 A$ ), the optimal age estimates were younger than expected. For example, when node A was constrained to be at least 33 mybp, the optimal ages of (the uncalibrated) nodes $\mathrm{B}$ and $\mathrm{C}$ were ca. 39 and 35 mybp, respectively. On the basis of fossil data for nodes B and C, they should be at least 43 and 49 mybp, respectively. Similarly, when node B was constrained to be at least 43 mybp, nodes $A$ and $C$ were inferred to be 32 and 34 mybp, respectively, which should be at least 33 and 49 mybp, respectively. We interpret this to mean that the best age estimates for the family should be based on all three calibration points (table $2 B, 2 C$ ). Results of age estimates from NPRS and PL are compared in figure 3, and the chronogram for PL with all calibration points enforced is shown in figure 2. The optimal smoothing value for the PL analyses (table 2C) was 316.23.

NPRS and PL analyses (table $2 B, 2 C$, respectively) indicate that Malpighiaceae originated during the latest Cretaceous (ca. 80-74 mybp, NPRS; 73-65 mybp, PL; Cretaceous time scale sensu Gradstein et al. 1995), which is consistent with previous age estimates based on NPRS (63.6 \pm 5.8 mybp; Davis et al. 2002b). There are a total of six disjunctions between New and Old World Malpighiaceae (fig. 2, nodes 1-6; table 2). The first disjunction occurred during the Maastrichtian (ca. 72-66 mybp, NPRS) or Paleocene (63-57 mybp, $\mathrm{PL})$, the second from middle Eocene to Oligocene (44-31 mybp, NPRS; 41-26 mybp, PL), the third during the late Eocene to earliest Miocene (34-24 mybp, NPRS) or late Eocene to late Oligocene (37-28 mybp, PL), the fourth during the middle Eocene to late Oligocene (46-26 mybp, NPRS; 40-25 mybp, PL), the fifth during the Oligocene to middle Miocene (29-11 mybp, NPRS; 26-16 mybp, PL), and the sixth during the late Oligocene to middle Miocene (26-11 mybp, NPRS) or earliest to middle Miocene (24-12 mybp, PL).

\section{Discussion}

\section{Dating Analyses}

Age estimates from NPRS and PL mostly overlapped (fig. 3 ), the exception being for the two deepest nodes in the tree (nodes 0 and 1). In these two cases, the age estimates from NPRS were older than those from PL. NPRS tends to overfit the data (Sanderson 2002), resulting in high levels of rate variation despite small changes in the data. Sanderson (2002) found that PL outperformed both the clock (Langley and Fitch 1974) and the nonclock method NPRS whenever data depart from a molecular clock. Thus, because our combined 
Table 2

Molecular Divergence Time Estimates based on NPRS $(A, B)$ and PL $(C)$

\begin{tabular}{|c|c|c|c|c|c|c|c|c|c|}
\hline \multicolumn{3}{|c|}{ Fossil calibration points } & \multirow{2}{*}{$\begin{array}{c}\begin{array}{c}\text { Malpigh } \\
\text { origin }\end{array} \\
0\end{array}$} & \multicolumn{6}{|c|}{ New World-Old World disjuncts } \\
\hline A & $\mathrm{B}$ & $\mathrm{C}$ & & 1 & 2 & 3 & 4 & 5 & 6 \\
\hline \multicolumn{10}{|c|}{ A. NPRS (individual calibration points) } \\
\hline 33 & $\begin{array}{c}38.73 \\
\quad(27.54 \\
48.08)\end{array}$ & $\begin{array}{c}34.73 \\
\quad(33.14 \\
39.04)\end{array}$ & $\begin{array}{c}66.57 \\
(62.05, \\
73.31)\end{array}$ & $\begin{array}{l}56.83 \\
\quad(52.89 \\
62.25)\end{array}$ & $\begin{array}{l}28.69 \\
(25.10 \\
33.00)\end{array}$ & $\begin{array}{l}20.64 \\
\quad(17.45 \\
24.42)\end{array}$ & $\begin{array}{l}29.19 \\
(21.89 \\
35.38)\end{array}$ & $\begin{array}{r}13.54 \\
(9.19 \\
18.84)\end{array}$ & $\begin{array}{l}12.92 \\
\quad(9.20 \\
17.23)\end{array}$ \\
\hline $\begin{array}{c}32.24 \\
(25.63 \\
37.76)\end{array}$ & 43 & $\begin{array}{c}34.12 \\
\quad(29.39 \\
39.48)\end{array}$ & $\begin{array}{l}67 \\
\quad(62.36, \\
73.76)\end{array}$ & $\begin{array}{l}57.05 \\
\quad(52.05 \\
62.66)\end{array}$ & $\begin{array}{l}28.01 \\
\quad(23.16 \\
33.42)\end{array}$ & $\begin{array}{l}20.25 \\
\quad(16.58 \\
24.74)\end{array}$ & $\begin{array}{l}28.93 \\
\quad(21.41 \\
35.83)\end{array}$ & $\begin{array}{c}13.31 \\
\quad(9.05 \\
19.73)\end{array}$ & $\begin{array}{l}12.68 \\
\quad(8.98, \\
17.48)\end{array}$ \\
\hline $\begin{array}{c}46.64 \\
\quad(41.60 \\
48.90)\end{array}$ & $\begin{array}{c}47.30 \\
(34.17 \\
65.18)\end{array}$ & 49 & $\begin{array}{c}76.88 \\
(74.33 \\
79.64)\end{array}$ & $\begin{array}{c}68.74 \\
\quad(65.99 \\
71.85)\end{array}$ & $\begin{array}{l}40.56 \\
\quad(29.85 \\
44.16)\end{array}$ & $\begin{array}{c}29.39 \\
\quad(23.61 \\
34.10)\end{array}$ & $\begin{array}{c}39.84 \\
\quad(26.73 \\
46.48)\end{array}$ & $\begin{array}{c}19.37 \\
\quad(12.31 \\
28.91)\end{array}$ & $\begin{array}{l}18.45 \\
\quad(12.15 \\
25.10)\end{array}$ \\
\hline \multicolumn{10}{|c|}{ B. NPRS (all calibration points included) } \\
\hline 33 & 43 & 49 & $\begin{array}{c}76.90 \\
\quad(74.02 \\
80.31)\end{array}$ & $\begin{array}{c}68.76 \\
(66.09 \\
71.79)\end{array}$ & $\begin{array}{c}40.59 \\
(31.43 \\
44.16)\end{array}$ & $\begin{array}{c}29.39 \\
\quad(23.60 \\
34.10)\end{array}$ & $\begin{array}{c}39.82 \\
(26.24 \\
46.46)\end{array}$ & $\begin{array}{c}19.49 \\
\quad(11.42 \\
28.80)\end{array}$ & $\begin{array}{l}18.45 \\
\quad(11.22, \\
25.92)\end{array}$ \\
\hline \multicolumn{10}{|c|}{ C. PL (all calibration points included) } \\
\hline 33 & 43 & 49 & $\begin{array}{l}68.58 \\
\quad(64.82, \\
72.69)\end{array}$ & $\begin{array}{l}59.85 \\
\quad(56.88 \\
63.33)\end{array}$ & $\begin{array}{l}33.78 \\
\quad(26.47 \\
41.25)\end{array}$ & $\begin{array}{c}32.37 \\
(27.88 \\
36.93)\end{array}$ & $\begin{array}{c}31.42 \\
(24.73 \\
39.61)\end{array}$ & $\begin{array}{l}20.90 \\
\quad(16.32 \\
26.06)\end{array}$ & $\begin{array}{r}17.40 \\
(11.83, \\
23.54)\end{array}$ \\
\hline
\end{tabular}

Note. Confidence intervals shown in parentheses following optimal ages estimates. Italicized ages in section $A$ indicate optimal age estimates that are younger than expected based on unenforced calibration points. Fossil calibration points (shown in bold) provide minimum age constraints for starred nodes labeled $A, B$, and $C$ in figure 2 (from Hably and Manchester 2000 [A], Taylor and Crepet 1987 [B], Jaramillo and Dilcher $2001[C])$. NPRS = nonparametric rate smoothing; PL = penalized likelihood.

$n d h \mathrm{~F}$ and PHYC data depart from a molecular clock, we based our reconstruction of the biogeographic history of Malpighiaceae on age estimates from PL (table 2C; fig. 2).

\section{Historical Biogeography}

Gondwanian aborigines and American colonists. Paleogeographic reconstructions of the breakup of western Gondwana suggest that direct land connections between Africa and South America were lost by the Upper Middle Cretaceous (ca. 105 mybp, sensu McLoughlin 2001), and by ca. 84 mybp, all plant migration between the remnants of western Gondwana likely ceased except through chance longdistance dispersal (Raven and Axelrod 1974). If transatlantic connections between New and Old World Malpighiaceae were consistent with western Gondwanan vicariance, we would expect the origin of the family, as well as relevant disjunctions, to have occurred before 84 mybp. Our Late Cretaceous age estimate (73-65 mybp; table 2C) for the origin of Malpighiaceae is more recent than this. Moreover, all of the disjunctions between New and Old World clades (table 2C; 63-12 mybp) are even younger, rendering the Gondwanian aborigine hypothesis (Vogel 1990; fig. 1A) untenable as an explanation of extant Malpighiaceae distribution.

Our age estimates are potentially consistent with a series of episodic long-distance dispersal events across the southern Atlantic (American colonist scenario; fig. 1B; Raven and Axelrod 1974; Anderson 1981, 1990). Under a strict dispersalist scenario, however, we would need to invoke at least six over- water dispersal events to explain the distribution of Malpighiaceae. Furthermore, these dispersals would have to be postulated for (1) times starting in the Paleocene (ca. 60 mybp), when the distance between Africa and South America was already substantial (Scotese et al. 1988), and (2) later in the Tertiary, when the continents were even further apart. Moreover, the American colonist scenario does not account for well-preserved Eocene and Oligocene fossils of Malpighiaceae from Hungary and Slovenia (Tetrapterys) and Tennessee (Eoglandulosa warmanensis), both of which suggest a role for Northern Hemisphere high-latitude intercontinental migration of at least some lineages of Malpighiaceae (see below).

It is possible that some of the six disjuncts do result from long-distance dispersal. The chance of long-distance dispersal increases dramatically over time (Simpson 1952), and the undeniable importance of this mode is illustrated by the terrestrial biota of the Hawaiian Islands (Carlquist 1966, 1974). The phylogenetic distribution of fruit types, however, suggests that long-distance transport in Malpighiaceae is rare. Fleshy bird-dispersed fruits have evolved in at least three New World Malpighiaceae (Anderson 1978; Davis et al. 2001), but none of these has become established in the Old World, despite their high diversity and widespread distribution (Anderson 1978). Rather, only Malpighiaceae with winged wind-dispersed fruits have become established in the Old World. In contrast to fleshy bird-dispersed fruits, winddispersed taxa constitute a very small percentage of $\mathrm{Ha}$ waiian Island seed plant immigrants (ca. $40 \%$ vs. $<1.5 \%$; 


\section{NPRS vs PL}

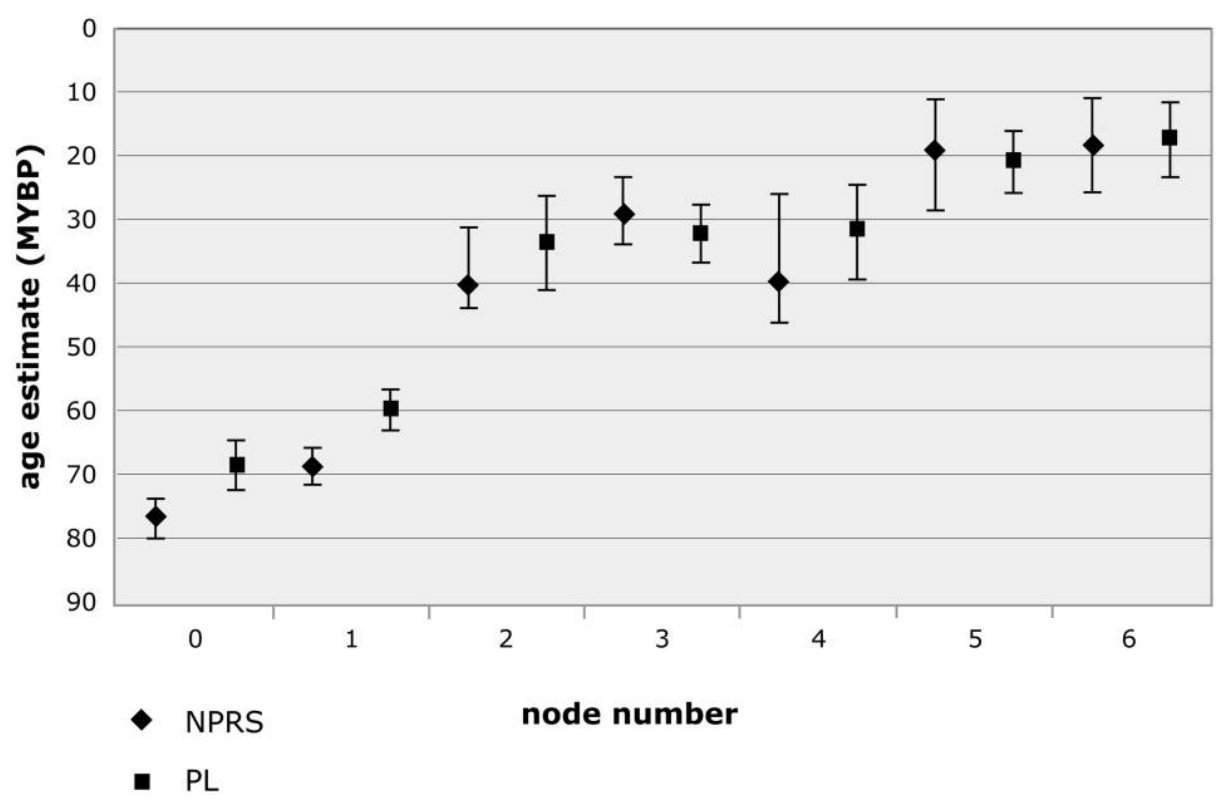

Fig. 3 Nonparametric rate smoothing (NPRS) versus penalized likelihood (PL). Optimal age estimates for nodes 0-6 shown with a diamond (NPRS) or square (PL). Confidence intervals around optimal ages estimated with the method of Cutler (2000) as implemented in r8s (Sanderson 2003).

Carlquist 1974, p. 77, table 2.1), suggesting that these fruits do not easily travel for long distances over water. Taken together, these data indicate that winged fruits might have facilitated travel across short oceanic barriers, such as those in the nascent Caribbean and across the North Atlantic but not across the distances that separated South America from Africa.

Northern Hemisphere migration. Migration across Northern Hemisphere land bridges has been considered a viable scenario for the intercontinental migration of thermophilic evergreen flowering plants that today inhabit tropical regions (Raven and Axelrod 1974; Wolfe 1975). Two Northern Hemisphere land connections, one spanning Beringia, the other spanning the North Atlantic, existed in the Tertiary. Beringia has played an important role for plant migrations throughout the Tertiary for temperate and boreal groups distributed across the Northern Hemisphere (Wolfe 1975; Tiffney 1985a, 1985b; Manchester 1999; Donoghue et al. 2001; Tiffney and Manchester 2001). Although our estimates of divergence times are compatible with overland migration via Beringia, its high latitude (between $69^{\circ}$ and $75^{\circ} \mathrm{N}$; Tiffney $1985 b$ ), limited light availability, and lower temperatures (Tiffney and Manchester 2001) probably restricted its use by broad-leaved evergreen thermophilic lineages for most, if not all, of the Tertiary. Furthermore, the geographic distribution of neither fossils nor extant Malphighiaceae supports this route for the dispersion of the family. Fossils are distributed between eastern North America, northern Europe, the Neotropics, Africa, and Australia. Extant Old World Malpighiaceae are almost entirely restricted to Africa and Madagascar, with a few, mostly minor, incursions into Asia
(Arènes 1957). If Malpighiaceae had utilized Beringia, we would expect fossil localities from eastern Asia or western North America (or both) and more family diversity in tropical Asia today.

The more likely Northern Hemisphere route for Malpighiaceae during the Tertiary is via the North Atlantic. The warmest climates in the Northern Hemisphere occurred in the late Paleocene/Eocene (Zachos et al. 2001), and midlatitude fossil floras from this time all contain a large percentage of thermophilic evergreen taxa (Wolfe 1978; Tiffney 1985a, 1985b, 2000; Manchester 1999; Tiffney and Manchester 2001; Jaramillo 2002). This period coincides with the presence of a land bridge spanning the North Atlantic during a time when the ocean was much narrower. This bridge extended south to roughly $45^{\circ} \mathrm{N}$ and is thought to have maintained sufficient warmth, light, and humidity for thermophilic broad-leaved evergreen plants to flourish (Tiffney and Manchester 2001). Beginning in the late Eocene, climates progressively cooled, and the potential for thermophilic evergreen elements across the North Atlantic diminished accordingly. The early Miocene warming period (Zachos et al. 2001) apparently supported mainly temperate and warm-temperate vegetation in the North Atlantic, giving way to cool-temperate and boreal vegetation by the middle Miocene. Although direct land connections were apparently severed by the middle Eocene, there was probably ample opportunity throughout the Tertiary for island hopping in the region (Tiffney and Manchester 2001).

The optimal estimates for the six disjunctions between New and Old World Malpighiaceae (table 2C) indicate transatlantic migration of the family during three major time 
intervals: late Paleocene (ca. 60 mybp; fig. 2, node 1), latest Eocene-earliest Oligocene (ca. 34-31 mybp; fig. 2, nodes 2-4), and early Miocene (ca. 21-17 mybp; fig. 2, nodes 5, 6). The time of disjunction at node 1 coincides with the dominance of the boreotropical flora and the North Atlantic Land Bridge; it can easily be accommodated by the North Atlantic corridor. Despite somewhat cooler climates in the Oligocene across the Northern Hemisphere, the occurrence of fossil Tetrapterys (a genus that today is exclusively New World) in the Oligocene of northern Europe strongly suggests that the North Atlantic remained a viable corridor for intercontinental migration of Malpighiaceae until at least this time; thus, nodes 2, 3, and 4 can also be accommodated by a North Atlantic migration. The times of disjunction of nodes 5 and 6 correspond to the early Miocene warming period. This period primarily supported mixed-mesophytic vegetation throughout the middle latitudes in the Northern Hemisphere, and it is unclear whether the North Atlantic could support thermophilic broad-leaved evergreen elements such as Malpighiaceae (Tiffney and Manchester 2001). The few postEocene floras that are known from the vicinity of the North Atlantic suggest an early Miocene warm-temperate vegetation and later cool-temperate and boreal vegetation. Thus, whether a North Atlantic migration or long-distance dispersal best accounts for these Miocene disjuncts remains an open question. A clearer picture of the boreotropical flora during the Miocene is needed before northern migration is ruled out entirely for these more recent disjuncts.

Based on the above, we offer the following scenario for the historical biogeography of Malpighiaceae. The Neotropical subfamily Byrsonimoideae (sensu Davis et al. 2001) is sister to all Malpighiaceae. Their phylogenetic placement and high diversity in northern South America indicates that Malpighiaceae may have originated in South America during the latest Cretaceous (ca. 69 mybp; table 2C) in isolation from Africa (see also Raven and Axelrod 1974; Anderson 1981, 1990; Davis et al. 2002b). From South America, these groups migrated into present-day North America across the Caribbean Basin, which consisted of scattered continental and volcanic islands (Iturralde-Vinent and MacPhee 1999). These connections appear to have been fairly direct between North and South America, starting during the late Eocene (ca. 35 mybp; MacPhee and Iturralde-Vinent 1995; Iturralde-Vinent and MacPhee 1999) and perhaps extended into the Miocene. Our data suggest that Malpighiaceae may have migrated into North America via the proto-Caribbean even earlier in the Tertiary, starting during the Paleocene (node 1; see also Davis et al. 2002a).

Once Malpighiaceae became established in North America, they were able to move across North Atlantic land routes (Tiffney 1985a, 1985b, 2000) during periods of global warming (Zachos et al. 2001). The subsequent movement of Malpighiaceae into Africa (and later into Madagascar) with minor incursions into Asia may be attributable to cooling during the Oligocene (Berggren and Prothero 1992; Tiffney and Manchester 2001; Zachos et al. 2001) that initiated the southward retreat and ultimately vicariance of thermophilic lineages (Collinson 1992; Wolfe 1992; Manchester 1999; Davis et al. 2002a, 2002b). Subsequent warming during the Miocene (Tiffney 1985b; Tiffney and Manchester 2001;
Zachos et al. 2001) may have resulted in further intercontinental migration across the North Atlantic, but long-distance dispersal of these Miocene disjuncts remains a viable alternative. More knowledge of North Atlantic paleoecology would help clarify the significance of divergence times for both thermophilic and mixed-mesophytic plant lineages that may be too recent for Northern Atlantic land bridges to accommodate (Xiang et al. 1998; Wen 1999; Donoghue et al. 2001; Tiffney and Manchester 2001).

Neotropical oil-bee pollinators. One potential problem with our hypothesis is that the primary pollinators of Malpighiaceae have not made it to the Old World, as would be expected if Northern Hemisphere migration (vs. longdistance dispersal) were a major factor in the amphi-Atlantic distribution of Malpighiaceae. Neotropical Malpighiaceae appear to have coevolved with their centridine (and tapinotaspidine) pollinators (Vogel 1974, 1990; Anderson 1979, 1990; Neff and Simpson 1981; Taylor and Crepet 1987). One might thus expect that the migration of Malpighiaceae would be limited by the distribution of their primary pollinators. However, unlike Malpighiaceae, these bees have not made it into the Old World (Vogel 1974, 1990; Michener 2000). Paleotropical Malpighiaceae instead exhibit a diverse array of floral morphologies and appear to be pollinated by a host of insects whose primary reward is pollen rather than oil (Vogel 1990; C. Davis, unpublished data). Unfortunately, there are no reliable fossils for these bees (M. Engel and C. Michener, personal communication), from which their ability to disperse along with Malpighiaceae might be inferred. However, several phylogenetically diverse Neotropical Malpighiaceae, along with most of the Old World taxa, have lost many (in some cases all) of the floral characteristics associated with oil-bee pollination (Anderson 1979; Vogel 1990; W. Anderson, personal communication; C. Davis, unpublished data). That they are apparently capable of existing outside of this selective regime, in both the New and Old Worlds, indicates that the migration of Malpighiaceae was not limited by the distribution of their primary pollinators. It may be that various ecological factors restricted the coincident migration of oil-pollinating bees that visit New World Malpighiaceae. An investigation of floral evolution in Malpighiaceae is ongoing by one of us (C. Davis) and may shed light on their association with these bees.

\section{Conclusions}

Our results, and those from several other studies, indicate that North Atlantic land connections may have played an important role not only in the development of the temperate biota for plants (Tiffney 1985a, 1985b; Wen 1999; Donoghue et al. 2001; Tiffney and Manchester 2001) and animals (Sanmartín et al. 2001) but also in the migration of tropical lineages no longer present at high latitudes. Dispersion via the North Atlantic may have been possible for Malpighiaceae starting in the Paleocene and extending into the early Oligocene, during periods when northern latitudes supported tropical forests. Our data also indicate, however, that the most recent disjunctions in Malpighiaceae occurred during the Miocene, when North Atlantic land connections may have been unavailable and cooler climates existed. Whether these 
Miocene disjuncts arose via Northern Hemisphere migration or long-distance dispersal awaits further paleontological and phylogenetic investigation.

\section{Acknowledgments}

A. Amorim, W. Anderson, J. Davis, D. Dilcher, M. Donoghue, J. Doyle, C. Jaramillo, R. Morley, J. Neff, R. Nyffeler, S. Renner, J. Rest, F. Rutschmann, M. Sanderson, and T. Scharaschkin provided assistance with this article. D. Min- dell and P. Tucker provided lab space in the Ruthven $\mathrm{Mu}$ seum. S. Manchester allowed C. Davis to study fossil Tetrapterys at the Florida Museum of Natural History. T. Givnish and S. Renner kindly invited us to contribute this article. Funding was provided to C. Davis by a Deland Award from the Arnold Arboretum, National Science Foundation Doctoral Dissertation Improvement grant DEB-0073299, and a Rackham Faculty grant from the University of Michigan. The Michigan Society of Fellows helped C. Davis complete this study. This article honors Dr. Robert Thorne.

\section{Literature Cited}

Anderson WR 1978 Byrsonimoideae, a new subfamily of the Malpighiaceae. Leandra 7:5-18.

1979 Floral conservatism in Neotropical Malpighiaceae. Biotropica 11:219-223.

- 1981 Malpighiaceae in B. Maguire, ed. The botany of the Guayana Highland. Pt XI. Mem NY Bot Gard 32:21-305.

1990 The origin of the Malpighiaceae: the evidence from morphology. Mem NY Bot Gard 64:210-224.

APG (Angiosperm Phylogeny Group) 2003 An update of the Angiosperm Phylogeny Group classification for the orders and families of flowering plants: APG II. Bot J Linn Soc 141:399-436.

Arènes J 1957 Répartition géographique des Malpighiacées vivantes et fossiles. 1. CR Somm Seances Soc Biogeogr 290:81-108.

Barthlott W 1983 Biogeography and evolution in Neo- and palaeotropical Rhipsalinae (Cactaceae). Pages 241-248 in K Kubitzki, ed. Dispersal and distribution. Parey, Hamburg.

Barthlott W, DR Hunt 1993 Cactaceae. Pages 161-197 in K Kubitzki, JG Rohwer, V Bittrich, eds. The families and genera of vascular plants. Vol 2. Springer, Berlin.

Barthlott W, NP Taylor 1995 Notes towards a monograph of Rhipsalideae (Cactaceae). Bradleya 13:43-79.

Berggren WA, DV Kent, CC Swisher, II, MP Aubry 1995 A revised Cenozoic geochronology and chronostratigraphy. Pages 129-212 in WA Berggren, DV Kent, MP Aubry, J Hardenbol, eds. Geochronology, time scales and global stratigraphic correlation. SEPM (Soc Sediment Geol), Tulsa, Okla.

Berggren WA, DR Prothero 1992 An overview. Pages 1-28 in DR Prothero, WA Berggren, eds. Eocene-Oligocene climatic and biotic evolution. Princeton University Press, Princeton, N.J.

Bremer K 1993 Intercontinental relationships of African and South American Asteraceae: a cladistic biogeographic analysis. Pages 105-135 in P Goldblatt, ed. Biological relationships between Africa and South America. Yale University Press, New Haven, Conn.

Carlquist S 1966 The biota of long-distance dispersal. II. Loss of dispersibility in Pacific Compositae. Evolution 20:30-48.

1974 Island biology. Columbia University Press, New York.

Chanderbali AS, H van der Werff, SS Renner 2001 Phylogeny and historical biogeography of Lauraceae: evidence from the chloroplast and nuclear genomes. Ann Mo Bot Gard 88:104-134.

Collinson ME 1992 Vegetational and floristic changes around the Eocene/Oligocene boundary in western and central Europe. Pages 437-450 in DR Prothero, WA Berggren, eds. Eocene-Oligocene climatic and biotic evolution. Princeton University Press, Princeton, N.J.

Cutler DJ 2000 Estimating divergence times in the presence of an overdispersed molecular clock. Mol Biol Evol 17:1647-1660.

Davis CC 2002 Madagasikaria (Malpighiaceae): a new genus from Madagascar with implications for floral evolution in Malpighiaceae. Am J Bot 89:699-706.

Davis CC, WR Anderson, MJ Donoghue 2001 Phylogeny of Mal- pighiaceae: evidence from chloroplast $n d h F$ and $t r n L-F$ nucleotide sequences. Am J Bot 88:1830-1846.

Davis CC, CD Bell, PW Fritsch, S Mathews 2002a Phylogeny of Acridocarpus-Brachylophon (Malpighiaceae): implications for Tertiary tropical floras and Afroasian biogeography. Evolution 56: 2395-2405.

Davis CC, CD Bell, S Mathews, MJ Donoghue $2002 b$ Laurasian migration explains Gondwanan disjunctions: evidence from Malpighiaceae. Proc Natl Acad Sci USA 99:6833-6837.

Davis CC, MW Chase 2004 Elatinaceae are sister to Malpighiaceae; Peridiscaceae belong to Saxifragales. Am J Bot 91:262-273.

Dilcher DL 1973 A paleoclimatic interpretation of the Eocene floras of southeastern North America. Pages 39-59 in A Graham, ed. Vegetation and vegetational history of northern Latin America. Elsevier, Amsterdam.

Donoghue MJ, CD Bell, J Li 2001 Phylogenetic patterns in Northern Hemisphere plant geography. Int J Plant Sci 162(suppl): S41-S52.

Doyle JA, MJ Donoghue 1993 Phylogenies and angiosperm diversification. Paleobiology 19:141-167.

Doyle JA, A Le Thomas 1997 Phylogeny and geographic history of Annonaceae. Geogr Phys Quatern 51:353-361.

Farris JS, M Källersjö, AG Kluge, C Bult 1994 Testing significance of incongruence. Cladistics 10:315-319.

Felsenstein J 1981 Evolutionary trees from DNA sequences: a maximum likelihood approach. J Mol Evol 17:368-376.

1985 Confidence limits on phylogenies: an approach using the bootstrap. Evolution 39:783-791.

Fitch WM 1971 Toward defining the course of evolution: minimum change for a specific tree topology. Syst Zool 20:406-416.

Frederiksen NO 1980 Mid-Tertiary climate of southeastern United States: the sporomorph evidence. J Paleontol 54:728-739.

Fritsch PW 1999 Phylogeny of Styrax based on morphological characters, with implications for biogeography and infrageneric classification. Syst Bot 24:356-378.

2001 Phylogeny and biogeography of the flowering plant genus Styrax (Styracaceae) based on chloroplast DNA restriction sites and DNA sequences of the internal transcribed spacer region. Mol Phylogenet Evol 19:387-408.

Gentry AH 1982 Neotropical floristic diversity: phytogeographical connections between Central and South America, Pleistocene climatic fluctuations, or an accident of the Andean orogeny? Ann Mo Bot Gard 69:557-593.

1993 Diversity and floristic composition of lowland tropical forest in Africa and South America. Pages 500-547 in P Goldblatt, ed. Biological relationships between Africa and South America. Yale University Press, New Haven, Conn.

Germeraad JH, CA Hopping, J Muller 1968 Palynology of Tertiary sediments from tropical areas. Rev Palaeobot Palynol 6:189-348. 
Gibson AC, PS Nobel 1986 The cactus primer. Harvard University Press, Cambridge, Mass.

Gradstein FM, FR Agterberg, JG Ogg, J Hardenbol, P van Veen, J Thierry, Z Huang 1995 A Triassic, Jurassic and Cretaceous time scale. Pages 95-126 in WA Berggren, DV Kent, MP Aubry, J Hardenbol, eds. Geochronology, time scales and global stratigraphic correlation. SEPM (Soc Sediment Geol), Tulsa, Okla.

Hably L, SR Manchester 2000 Fruits of Tetrapterys (Malpighiaceae) from the Oligocene of Hungary and Slovenia. Rev Palaeobot Palynol 111:93-101.

Hershkovitz MA, EA Zimmer 1997 On the evolutionary origins of the cacti. Taxon 46:217-232.

Holmgren PK, NH Holmgren, LC Barnett 1990 Index herbariorum. Pt 1 . The herbaria of the world. New York Botanical Garden, New York.

Huelsenbeck JP, B Rannala 1997 Phylogenetic methods come of age: testing hypotheses in an evolutionary context. Science 276:227-232.

Huelsenbeck JP, F Ronquist 2001 MRBAYES: Bayesian inference of phylogenetic trees. Bioinformatics 17:754-755.

Iturralde-Vinent MA, RDE MacPhee 1999 Paleogeography of the Caribbean region: implications for Cenozoic biogeography. Bull Am Mus Nat Hist 238:1-95.

Jaramillo CA 2002 Response of tropical vegetation to Paleogene warming. Paleobiology 28:222-243.

Jaramillo CA, DL Dilcher 2001 Middle Paleogene palynology of Central Colombia, South America: a study of pollen and spores from tropical latitudes. Palaeontogr Abt B Palaeophytol 285:87-213.

Krutzsch W 1989 Paleogeography and historical phytogeography (paleochronology) in the Neophyticum. Plant Syst Evol 162:5-61.

Langley CH, WM Fitch 1974 An estimation of the constancy of the rate of molecular evolution. J Mol Evol 3:161-177.

Lavin M, M Luckow 1993 Origins and relationships of tropical North America in the context of the boreotropics hypothesis. Am J Bot 80:1-14.

Lavin M, M Thulin, J-N Labat, RT Pennington 2000 Africa, the odd man out: molecular biogeography of dalbergioid legumes (Fabaceae) suggests otherwise. Syst Bot 25:449-467.

Lowrie SR 1982 The palynology of the Malpighiaceae and its contribution to family systematics. PhD diss. University of Michigan, Ann Arbor. (University Microfilms \#82-24999.)

MacPhee RDE, MA Iturralde-Vinent 1995 Origin of the greater Antillean land mammal fauna. 1. New Tertiary fossils from Cuba and Puerto Rico. Am Mus Novit 3141:1-31.

Magallón S, PR Crane, PS Herendeen 1999 Phylogenetic pattern, diversity, and diversification of eudicots. Ann Mo Bot Gard 86: 297-372.

Magallón S, MJ Sanderson 2001 Absolute diversification rates in angiosperm clades. Evolution 55:1762-1780.

Manchester SR 1999 Biogeographical relationships of North American Tertiary floras. Ann Mo Bot Gard 86:472-522.

Martin HA 2002 History of the family Malpighiaceae in Australia and its biogeographic implications: evidence from pollen. Aust J Bot 50:171-182.

McLoughlin S 2001 The breakup history of Gondwana and its impact on pre-Cenozoic floristic provincialism. Aust J Bot 49: 271-300.

Michener CD 2000 The bees of the world. Johns Hopkins University Press, Baltimore.

Muller J 1981 Fossil pollen records of extant angiosperms. Bot Rev (Lancaster) 47:1-142.

Neff JL, BB Simpson 1981 Oil-collecting structures in the Anthophoridae (Hymenoptera): morphology, function and use in systematics. J Kans Entomol Soc 54:95-123.

Nyffeler R 2002 Phylogenetic relationships in the cactus family
(Cactaceae) based on evidence from trnK/matK and trnL-trnF sequences. Am J Bot 89:312-326.

Posada D, KA Crandall 1998 MODELTEST: testing the model of DNA substitution. Bioinformatics 14:817-818.

Potter FW Jr, DL Dilcher 1980 Biostratigraphic analysis of Eocene clay deposits in Henry County, Tennessee. Pages 211-225 in DL Dilcher, TN Taylor, eds. Biostratigraphy of fossil plants. Dowden, Hutchinson \& Ross, Stroudsburg, Pa.

Raven PH, DI Axelrod 1974 Angiosperm biogeography and past continental movements. Ann Mo Bot Gard 61:539-673.

Regali M, N Uesugui, A Santos 1974 Palinología dos sedimentos Meso-Cenozoicos do Brasil. Bol Tec Petrobras 17:177-191.

Renner SS, G Clausing, K Meyer 2001 Historical biogeography of Melastomataceae: the roles of Tertiary migration and long-distance dispersal. Am J Bot 88:1290-1300.

Rodríguez F, JF Oliver, A Marín, JR Medina 1990 The general stochastic model of nucleotide substitution. J Theor Biol 142: 485-501.

Rull V 1997 Sequence analysis of western Venezuelan Cretaceous to Eocene sediments using palynology: chrono-paleoenvironmental and paleovegetational approaches. Palynology 21:79-90.

1998 Middle Eocene mangroves and vegetation changes in the Maracaibo Basin, Venezuela. Palaios 13:287-296.

Salard-Cheboldaeff M 1991 Intertropical African palynostratigraphy from Cretaceous to late Quaternary times. J Afr Earth Sci 11:1-24.

Sanderson MJ 1997 A nonparametric approach to estimating divergence times in the absence of rate constancy. Mol Biol Evol 14: $1218-1231$.

1998 Estimating rates and time in molecular phylogenies: beyond the molecular clock? Pages 242-264 in DE Soltis, PS Soltis, JA Doyle, eds. Molecular systematics of plants II: DNA sequencing. Kluwer Academic, Boston.

- 2002 Estimating absolute rates of molecular evolution and divergence times: a penalized likelihood approach. Mol Biol Evol 19:101-109.

2003 r8s: inferring absolute rates of molecular evolution and divergence times in the absence of a molecular clock. Bioinformatics 19:301-302.

Sanmartín I, H Enghoff, F Ronquist 2001 Patterns of animal dispersal, vicariance and diversification in the Holarctic. Biol J Linn Soc 73:345-390.

Scotese CR, LM Gahagan, RL Larson 1988 Plate tectonic reconstructions of the Cretaceous and Cenozoic ocean basins. Tectonophysics 155:27-48.

Simpson GG 1952 Probabilities of dispersal in geologic time. Bull Am Mus Nat Hist 99:163-176.

Smith AC 1973 Angiosperm evolution and the relationship of the floras of Africa and America. Pages 49-61 in BJ Meggers, ES Ayensu, WD Duckworth, eds. Tropical forest ecosystems in Africa and South America: a comparative review. Smithsonian Institution, Washington, D.C.

Soltis DE, AE Senters, MJ Zanis, S Kim, JD Thompson, PS Soltis, LP Ronse Decraene, PK Endress, JS Farris 2003 Gunnerales are sister to other core eudicots: implications for the evolution of pentamery. Am J Bot 90:461-470.

Swofford DL 2002 PAUP*: phylogenetic analysis using parsimony (" and other methods), version 4. Sinauer, Sunderland, Mass.

Taylor DW, WL Crepet 1987 Fossil floral evidence of Malpighiaceae and an early plant-pollinator relationship. Am J Bot 74:274-286.

Thorne RF 1972 Major disjunctions in the geographic ranges of seed plants. Q Rev Biol 47:365-411.

1973 Floristic relationships between tropical Africa and tropical America. Pages 27-47 in BJ Meggers, ES Ayensu, WD Duckworth, eds. Tropical forest ecosystems in Africa and South 
America: a comparative review. Smithsonian Institution, Washington, D.C.

Tiffney BH 1985a The Eocene North Atlantic land bridge: its importance in Tertiary and modern phytogeography of the Northern Hemisphere. J Arnold Arbor Harv Univ 66:243-273.

$1985 b$ Perspectives on the origin of the floristic similarity between eastern Asia and eastern North America. J Arnold Arbor Harv Univ 66:73-94.

2000 Geographic and climatic influences on the Cretaceous and Tertiary history of Euramerican floristic similarity. Acta Univ Carol Geol 44:5-16.

Tiffney BH, SR Manchester 2001 The use of geological and paleontological evidence in evaluating plant phylogenetic hypotheses in the Northern Hemisphere Tertiary. Int J Plant Sci 162(suppl): S3-S17.

Vogel S 1974 Ölblumen und ölsammelnde Bienen. Trop Subtrop Pflanzenwelt 7:283-547.

1990 History of the Malpighiaceae in the light of pollination ecology. Mem NY Bot Gard 55:130-142.

Wen J 1999 Evolution of eastern Asian and eastern North American disjunct distributions in flowering plants. Annu Rev Ecol Syst 30: 421-455.

Wikström N, V Savolainen, MW Chase 2001 Evolution of the angiosperms: calibrating the family tree. Proc R Soc Lond B Biol Sci 268:2211-2220.

Wolfe JA 1975 Some aspects of plant geography of the Northern Hemisphere during the late Cretaceous and Tertiary. Ann Mo Bot Gard 62:264-279.

1978 A paleobotanical interpretation of Tertiary climates in the Northern Hemisphere. Am Sci 66:694-703.

1992 Climatic, floristic, and vegetational changes near the Eocene/Oligocene boundary in North America. Pages 421-436 in DR Prothero, WA Berggren, eds. Eocene-Oligocene climatic and biotic evolution. Princeton University Press, Princeton, N.J.

Xiang Q-Y, DE Soltis, PS Soltis 1998 The eastern Asian and eastern and western North American floristic disjunction: congruent phylogenetic patterns in seven diverse genera. Mol Phylogenet Evol 10:178-190.

Zachos J, M Pagani, L Sloan, E Thomas, K Billups 2001 Trends, rhythms, and aberrations in global climate $65 \mathrm{Ma}$ to present. Science 292:686-693. 